Artigo

\title{
Determinação de Regiões Homogêneas do Índice de Precipitação Normalizada (SPI) na Amazônia Oriental
}

\author{
Sergio Rodrigo Quadros dos Santos ${ }^{1}$, Célia Campos Braga ${ }^{2}$, Clovis Angeli Sansigolo ${ }^{1}$, \\ Ana Paula Paes dos Santos ${ }^{1}$ \\ ${ }^{1}$ Centro de Previsão de Tempo e Estudos Climáticos, Instituto Nacional de Pesquisas Espaciais, \\ São José dos Campos, SP, Brasil. \\ ${ }^{2}$ Universidade Federal de Campina Grande, Departamento de Ciências Atmosféricas, \\ Campina Grande, PB, Brasil.
}

Recebido: 3 de Fevereiro de 2016-- Aceito: 15 de Maio de 2016

\begin{abstract}
Resumo
O presente estudo objetiva determinar Regiões homogêneas do SPI-6 na Amazônia Oriental e analisar as principais características dos eventos secos e chuvosos em cada região. Para o cálculo do SPI, utilizou-se dados de precipitação de 72 postos pluviométricos distribuídos na Amazônia Oriental. Além disso, aplicou-se o Agrupamento Hierárquico de Ward para determinar as Regiões Homogêneas e a Análise Fatorial em Componentes Principais (ACP) para determinar os principais padrões de variabilidade climáticas associados aos eventos secos e chuvosos. Os resultados obtidos pela Análise de Agrupamento mostraram a existência de três Regiões Homogêneas em relação ao SPI-6: RH-1 (litoral), RH-2 (central) e RH-3 (sul). De modo geral a região do litoral apresentou mais eventos secos que chuvosos. Nas demais regiões (central e sul) a situação é inversa, isto é, existem mais eventos de chuva, porém com pouca duração. Por meio da ACP verificou-se que os eventos secos e chuvosos na Amazônia Oriental estavam associados, principalmente, a variabilidade climática dos oceanos Atlântico (Gradiente do Atlântico) e Pacífico (ENOS).

Palavras-chave: Amazônia, precipitação, regiões homogêneas, SPI, ACP.
\end{abstract}

\section{Determination of Homogeneous Precipitation Regions of the Standardized Precipitation Index (SPI) in the Eastern Amazon}

\begin{abstract}
This paper aims to determine homogeneous regions of the SPI-6 in the Eastern Amazon and analyze the main characteristics of dry and rainy events in each region. For the calculation of the SPI, we used rainfall data of 72 rainfall stations distributed in the eastern Amazon. Furthermore, were used Ward hierarchical grouping to identify homogeneous regions and the Principal Components Factor Analysis (PCA) to determine the main climate variability patterns associated with dry and rainy events. The results showed three homogeneous regions (HR): HR-1 (coast), HR-2 (central region) and HR-3 (south). Generally, the coastal region showed more dry events than rain. In the other regions (central and south) the situation is reversed, i.e, there are more rain events, but with short duration. The results obtained by PCA showed that the dry and wet events in Eastern Amazonia were associated, mainly, to climate variability associated the Atlantic Ocean (Atlantic Dipole) and Pacific Ocean (ENSO).
\end{abstract}

Keywords: Amazon, rainfall, homogeneous regions, SPI, PCA.

\section{Introdução}

Estudos mostram que o Brasil é vulnerável às mudanças climáticas, especialmente quanto aos extremos climáticos. As áreas mais vulneráveis compreendem a Amazônia e o Nordeste do Brasil (Marengo, 2007; Ambrizzi et al.,
2007; Marengo et al., 2007). Os eventos extremos de chuva causam diversos prejuízos a sociedade, como as inundações, que provoca a destruição de casas e estradas. Já os eventos extremos de seca tem grande impacto no setor de energia elétrica, bem como no sistema de transporte e pesca

Autor de correspondência: Sergio Rodrigo Quadros dos Santos, sergiosanntos@yahoo.com.br. 
na Região Amazônica (Cavalcanti e Kousky, 2004; Cox et al., 2008; Ning Zeng et al., 2008; Marengo et al., 2008; Marengo et al., 2011; Tomasella et al., 2011; Marengo, 2012; Cavalcanti, 2012; Santos et al., 2012; Coelho et al., 2012; PBMC, 2013; Santos, 2014). Além disso, o conhecimento sobre possíveis cenários climático-hidrológicos futuros e as suas incertezas pode ajudar a estimar demandas de água no futuro e também a definir políticas ambientais de uso e gerenciamento de água para o futuro.

A variabilidade espacial e temporal da precipitação, e consequentemente dos extremos de chuva e seca, na Região Amazônica tem como moduladores principais os oceanos Atlântico Tropical e Pacífico Tropical. Alguns eventos extremos de seca na Amazônia foram relacionadas com eventos de El Niño, como em 1912, 1926, 1983 e 19971998 (Aceituno, 1988; Uvo et al., 1998; Williams et al., 2005). Entretanto, as secas extremas que ocorreram em 1964 e 2005 não foram relacionados com El Niño, indicando a influência da anomalia de temperatura da superfície do mar (TSM) do Oceano Atlântico Tropical na modulação destes eventos (Marengo et al., 2008; Zeng et al., 2008). A seca extrema de 2010 foi relacionado às ocorrências sucessivas do fenômeno El Niño no verão austral, e o aquecimento anômalo do Oceano Atlântico Tropical Norte na primavera e verão boreal (Espinoza et al., 2011; Marengo et al., 2011).

Todavia, eventos extremos de chuva foram associados ao fenômeno La Niña (e.g, 1989, 1999, 2009, 2011 e 2012) (Marengo et al., 2013; Espinoza et al., 2014). Por sua vez, o evento extremo de chuva observado em 2009 estava relacionado com o aquecimento anômalo do Oceano Atlântico Tropical Sul (Marengo et al., 2013). Os extremos de chuva que ocorreram no verão de 2014 e 2015 no sudoeste da Amazônia elevaram os níveis médios dos rios, causaram inundações em cidades, destruíram fazendas e estradas, prejudicaram o sistemas de pescas e isolaram alguns moradores. O estudo de Espinoza et al. (2014) sugere que as TSM no Atlântico Tropical Sul e Atlântico Subtropical foram um dos principais responsaveis pelo o transporte de umidade do Atlântico em direção a Amazônia, sendo excepcionalmente intenso durante o verão de 2014 e 2015.

$\mathrm{Na}$ tentativa de quantificar e caracterizar os eventos extremos de seca e chuva nas diferentes regiões do globo, foram criados índices que fossem capazes de identificar tais eventos. McKee et al. (1993) propuseram o Índice de Precipitação Normalizada (SPI), para quantificar o déficit de precipitação em diversas escalas de tempo. A versatilidade do SPI está na simplicidade do seu cálculo e na eficácia de identificar eventos secos e chuvosos em diferentes escalas de tempo $(3,6,12$ meses, etc), que auxilia no monitoramento da dinâmica temporal desses eventos, isto é, o desenvolvimento e declínio. Como é um índice padronizado a classificação das categorias de seca/chuva pode ser utilizada em qualquer região do globo (Hayes, 2000). Adicionalmente, o SPI apresenta uma vantagem em relação aos demais índices, como por exemplo, o Índice de Severidade de Seca de Palmer (PDSI), pois utiliza apenas dados mensais de precipitação, ou seja, não se faz necessário a utilização de outras variáveis meteorológicas na detecção dos eventos secos e chuvosos (Guttman, 1998). Criado nos Estados Unidos da América (EUA), o SPI tem sido empregado com frequência por pesquisadores em todo mundo (Bonaccorso et al., 2003; Sonmez et al., 2005; Michaelides e Pashiardis, 2008; Zhang et al., 2009; Sadeghi e Shamseldin, 2014).

Diante do exposto, o objetivo do presente estudo é determinar regiões homogêneas do SPI-6 na Amazônia Oriental e analisar as principais características (início, fim e duração) dos períodos secos e chuvoso em cada região homogêneas. Este estudo propiciará um melhor entendimento dos eventos secos e chuvosos na Região, disponibilizando informações científicas a comunidade em geral, sobre quais regiões da Amazônia Oriental merecem maior atenção por parte dos governantes, no sentido de amenizar as consequências geradas pelas intempéries do tempo.

\section{Material e Métodos}

\section{1. Área de estudo e dados}

Para o desenvolvimento deste trabalho, foram utilizados dois conjuntos de dados mensais de precipitação para o cálculo do SPI. O primeiro conjunto é composto por dados pluviômetrícos mensais de oito (8) estações automáticas do Instituto Nacional de Meteorologia (INMET), o segundo conjunto de dados mensais pluviométricos contém sessenta e quatro (64) estações automáticas da Agencia Nacional das águas (ANA), totalizando desta forma setenta e duas (72) estações, distribuídas no estado do Pará para o período de 32 anos (1980 a 2011) (Fig. 1). Em adição, utilizou-se estas 72 estações pois elas não apresentaram falhas e/ou falta de dados em sua série temporal de precipitação.

Utilizou-se também dados de temperatura da superfície do mar (TSM) do Climate Prediction Center/National Centers for Environmental Prediction (CPC/NCEP) que estão projetados em uma grade regular de $2,5^{\circ}$ de latitude $\mathrm{x}$ $2,5^{\circ}$ de longitude, com o propósito de se investigar a influência dos padrões observados sobre a superfície oceânica do Atlântico Tropical e Pacífico Equatorial nos eventos secos e chuvosos da Região em estudo.

\subsection{Metodologias}

\subsection{1. Índice de precipitação normalizada (SPI)}

O cálculo do SPI se inicia determinando uma função de densidade de probabilidade que descreve as séries temporais. A distribuição gama possui bom ajuste para variáveis contínuas que tenham limite inferior igual a zero e não possuem limite superior, por isso, é largamente utilizada para o estudo de séries históricas de precipitação (Wilks, 2006). A função gama é dada por: 


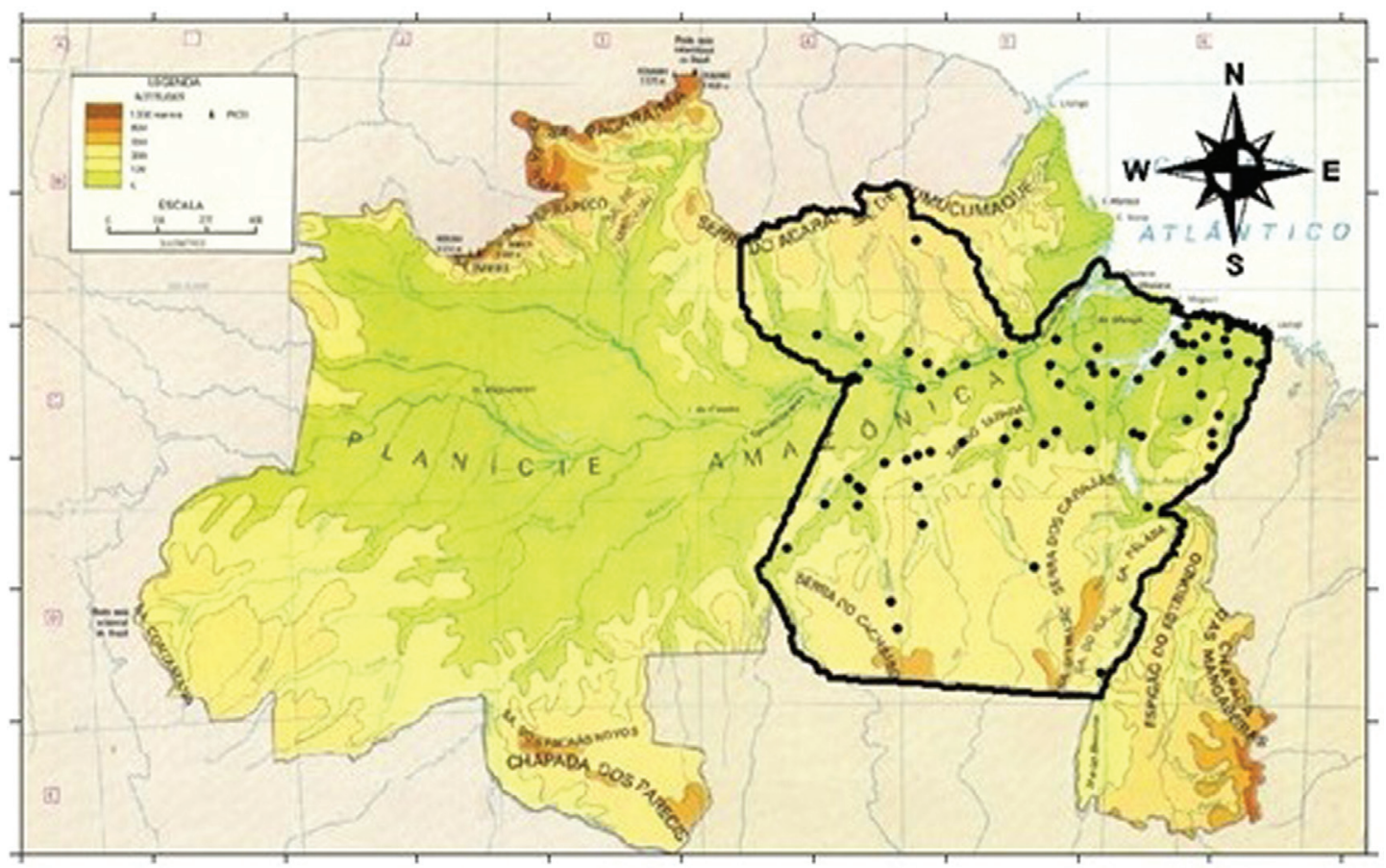

Figura 1 - Mapa de relevo e hidrografia do Norte do Brasil. Em detalhe a espacialização das estações pluviométricas na Amazônia Oriental. Fonte:Adaptado de Simielli (1991).

$$
g(x)=\frac{X^{\alpha-1} * e^{\frac{-x}{\beta}}}{\beta^{\alpha} \Gamma(\alpha)} \text { para } X>0
$$

Sendo, $\alpha>0$ parâmetro de forma; $\beta>0$ parâmetro de escala; $\mathrm{x}>0$, a quantidade de precipitação $(\mathrm{mm})$ e $\Gamma(\alpha)$ a função gama completa.

Para estimativa dos parâmetros $\alpha$ e $\beta$ da distribuição gama utilizou-se o método de

$$
\alpha=\frac{1}{4 A}\left(1+\sqrt{1+\frac{4 A}{3}}\right) \mathrm{e} \hat{\beta}=\frac{\bar{x}}{\alpha},
$$

onde o $\bar{x}$ é a precipitação média.

$$
A=\ln (\bar{x})-\frac{\sum \ln (x)}{n},
$$

onde o n é número de observações.

Deste modo a distribuição cumulativa é então transformada em distribuição probabilidade normal com média igual a zero e desvio padrão igual a um. Em seguida a probabilidade acumulada de ocorrência de cada valor mensal é estimada. Aplica-se a essa probabilidade a função normal inversa para encontrar o valor do SPI. O SPI nada mais é que a diferença da precipitação observada menos a média do intervalo de tempo específico, dividida pelo desvio padrão.

$$
S P I=Z_{i}=\frac{\left(P_{i}-\bar{P}_{i}\right)}{\sigma_{i}}
$$

Em que, $P_{i}$ é a precipitação observada; $\bar{P}_{i}$ e $\sigma_{\mathrm{i}}$, são respectivamente, a média e o desvio padrão da série ajustada. Finalmente, os valores encontrados foram classificados conforme McKee et al. (1993) (Tabela 1). Foram caracterizados não somente as secas, mas também os períodos chuvosos. Esse método tem a grande vantagem de padronizar a análise, permitindo comparar regiões totalmente distintas, como por exemplo, regiões com climas mais úmidos com regiões mais áridas.

O conceito de estações do ano para a Região Norte do Brasil é diferente das demais Regiões do Brasil. Em geral, tem-se a "estação seca" (junho a novembro) e a "estação chuvosa" (dezembro a maio) (Dos Santos et al., 2011; Lopes et al., 2013). Neste sentindo, utilizou-se a escala

Tabela 1 - Classificação dos períodos secos e chuvosos do SPI segundo McKee et al. (1993).

\begin{tabular}{lc}
\hline Valores de SPI & Classes \\
\hline$<-2,0$ & Seca Extrema \\
$-1,99$ a $-1,50$ & Seca Severa \\
$-1,49$ a $-1,0$ & Seca Moderada \\
$-0,99$ a $-0,49$ & Seca Leve \\
$-0,49$ a 0,49 & Quase Normal \\
0,49 a 0,99 & Chuva Leve \\
0,99 a 1,49 & Chuva Moderada \\
1,49 a 1,99 & Chuva Severa \\
$>2,0$ & Chuva Extrema \\
\hline
\end{tabular}


semestral do SPI (SPI-6) uma vez que essa escala representam os eventos de média duração, sendo útil para o monitoramento do período de transição entre estação seca e a chuvosa (Sansigolo, 2004 ; Dos Santos et al., 2014).

\subsubsection{Análise fatorial em componentes principais (ACP)}

A Análise de Componentes Principais é um procedimento matemático que usa uma transformação ortogonal para converter um conjunto de variáveis possivelmente correlacionadas, em um conjunto de valores de variáveis não correlacionadas, chamadas componentes principais (CPs). A principal vantagem da ACP perante as demais técnicas é porque possibilita determinar padrões espaciais e temporais consistentes e detalhados de variáveis meteorológicas (Wilks, 2006; Dos Santos et al., 2014; Rao et al., 2014).

Dependo dos dados a serem analisados, a ACP pode ser aplicada de diferentes modos. Neste estudo foi utilizado o modo S (S-mode factor analysis) o qual está relacionado com a análise de séries temporais correspondentes a pontos no espaço. Isto significa que a variável estatística analisada corresponde a séries temporais de uma variável meteorológica que pode ser considerada contínua no espaço e no tempo (Amanajás e Braga, 2012). Para aplicação desta técnica neste trabalho, organizou-se uma matriz de dados $\mathrm{M}(n \times p)$, onde as $n$ linhas correspondem aos meses, entre janeiro de 1980 a dezembro de 2011, e as $p$ colunas correspondem aos 72 postos pluviométrico.

A ACP em Modo-S produz componentes principais que são séries temporais (escores fatoriais em Modo-S). A carga fatorial para cada modo é obtida das correlações entre cada variável original e cada componente principal (Richman, 1986), constituindo- em mapas de coeficientes de correlação temporal. Valores de carga fatorial próximos a 1 representam padrões espaciais e temporais do SPI-6 similares aos fatores obtidos. Além dessa análise, verificou-se também, por meio da correlação entre o escore fatorial (série temporal) da primeira componente do SPI-6 e a série espacial das anomalias de TSM, a influência remota dos oceanos adjacentes nos eventos secos e chuvosos do SPI-6.

\subsubsection{Determinação das regiões homogêneas do SPI-6}

A determinação das regiões homogêneas do SPI-6 foi obtida pela classificação hierárquica proposta por Ward (1963), a partir dos 16 fatores comuns espaciais do SPI. Essa técnica objetiva descobrir a natureza complexa das relações multivariadas entre os dados investigados. A classificação das variáveis em grupos homogêneos e a identificação de suas características comuns possibilitam um melhor conhecimento da variável estudada (Gong e Richman, 1995).

Nos métodos aglomerativos, todos os processos de hierarquização são similares, iniciando pela determinação da função de agrupamento que é usada como critério para medir a distância entre dois indivíduos ou para estabelecer o quanto eles são similares. Para medir a similaridade ou dissimilaridade dos grupos, utilizou-se à distância euclidiana, a qual é dada como a raiz quadrada do somatório das diferenças entre os valores de $i$ e $j$ para todas as variáveis $(v=1, \ldots, p)$, expressa por:

$$
d_{i j}=\sqrt{\sum_{v=1}^{p}\left(x_{i v}-x_{j v}\right)^{2}}
$$

No caso dos métodos de hierarquização, os grupos são determinados por cortes transversais no dendrograma a partir do critério de agregação e do conhecimento prévio da região estudada (Everitt, 1993). Obtidas as Regiões Homogêneas do SPI-6, selecionou-se de maneira aleatória uma estação meteorológica, dentro de cada região homogênea, para realizar a análise dos aspectos climáticos do SPI-6, onde esta estação representa a região homogênea em que está inserida.

\section{Resultados}

\subsection{Padrões de variabilidade mensal do SPI- 6}

A aplicação da ACP aos valores mensais do SPI-6 resultou na obtenção dos fatores espaciais (Modo-S), dos quais foram obtidos 16 fatores, que explicaram aproximadamente $75 \%$ da variância total dos dados conforme, critério sugerido por Kaiser (North et al., 1982). Contudo, apenas o primeiro fator não-rotacionado foi analisado, pois ele explica 36,68\% da variância total dos dados (Tabela 2). Observa-se na Tabela 2 que, a partir do terceiro fator os autovalores decrescem lentamente, distanciando bastante dos modos subsequentes. Embora se tenham analisados

Tabela 2 - Componente principal (CP), Valores próprios, Variância explicada (\%) e Variância acumulada (\%) do SPI-6.

\begin{tabular}{lccc}
\hline CP & Valor próprio & Variância explicada & Variância acumulada \\
\hline 1 & 26,41 & 36,68 & 36,68 \\
2 & 3,95 & 5,49 & 42,17 \\
3 & 2,95 & 4,09 & 46,27 \\
4 & 2,82 & 3,91 & 50,18 \\
5 & 2,40 & 3,33 & 53,51 \\
6 & 2,12 & 2,94 & 56,45 \\
7 & 1,82 & 2,53 & 58,97 \\
8 & 1,78 & 2,47 & 61,44 \\
9 & 1,62 & 2,26 & 63,70 \\
10 & 1,51 & 2,10 & 65,80 \\
11 & 1,33 & 1,85 & 67,66 \\
12 & 1,29 & 1,80 & 69,45 \\
13 & 1,21 & 1,68 & 71,13 \\
14 & 1,14 & 1,58 & 72,71 \\
15 & 1,08 & 1,50 & 74,22 \\
16 & 1,02 & 1,42 & 75,63 \\
\hline
\end{tabular}


alguns fatores de pequena contribuição, só está sendo mostrada a discussão do padrão do primeiro fator, já que ele representa fisicamente a climatológica da Região.

O padrão espacial associado ao primeiro fator do SPI-6, que explica 36,68\% da variância total dos dados, apresenta correlações (cargas) superiores a 0,5 no noroeste e nordeste do Estado do Pará. Todavia, na ilha do Marajó (localizada ao nordeste do Estado) observa-se correlações inferiores a 0,5 , sugerindo a hipótese de que os fatores que geram precipitação nesta região possuem um caráter mais local. No sul do Estado do Pará, as correlações são ainda menores. De modo geral, a parte norte do Estado está mais sujeita a eventos de chuva que a parte sul do Estado (Fig. 2a). Isto ocorre devido o norte do Estado, além de ser influenciado pela atuação da ZCIT no período chuvoso, ao longo de todo o ano sofre influência da brisa oceânica, favorecendo a formação e propagação de sistemas convectivos, como as linhas de instabilidades (LI). Já o sul do Pará é influenciado principalmente pela ZCAS, durante a estação chuvosa (Amanajás e Braga, 2012; Dos Santos et al., 2014).

Adicionalmente, a série temporal associada ao primeiro fator espacial do SPI-6 evidência a alta variabilidade dos eventos secos e chuvosos do SPI-6 no Estado do Pará, isto é, valores positivos e negativos dos escores fatoriais alternando-se durante o período estudado. Além disso, a alternância dos escores fatorias evidência também que períodos secos (escores negativos) ocorreram dentro da estação chuvosa (dezembro a maio) da Região, assim como períodos chuvosos (escores positivos) ocorreram dentro da estação menos chuvosa (junho a novembro) (Fig. 2b).
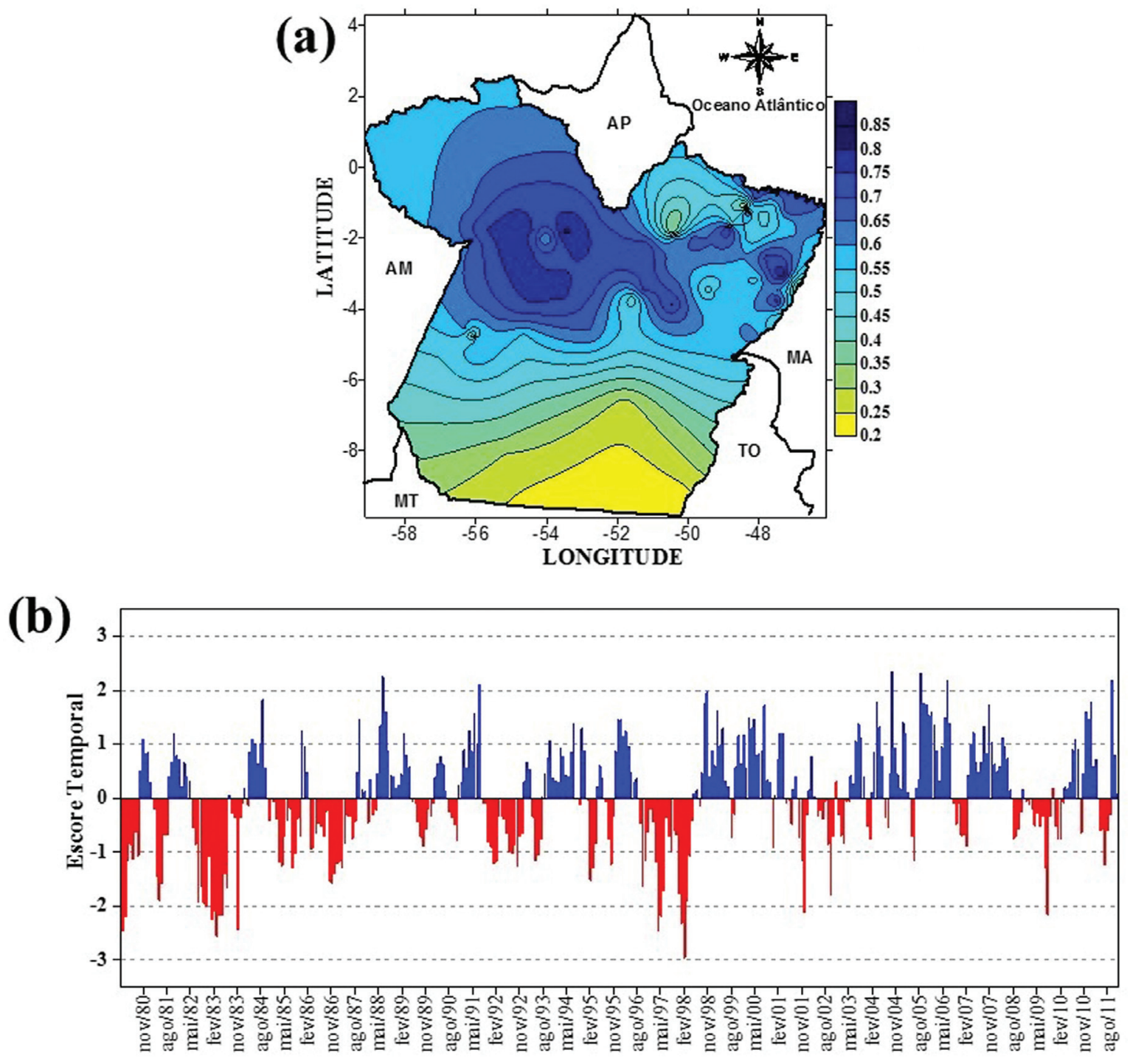

\section{Tempo}

Figura 2 - (a) Padrão espacial e (b) série temporal do primeiro fator espacial do SPI-6 obtidos a partir da ACP, compreendendo o período de junho de 1980 a dezembro de 2011. 
A Fig. 3 apresenta a correlação entre a série temporal do primeiro fator espacial do SPI-6 no Estado do Pará com as anomalias de TSM. Observa-se correlação positiva com as anomalias de TSM do Atlântico Tropical e correlação negativa com as anomalias de TSM do Pacífico Tropical, isto indica que os eventos chuvosos (secos) sobre o Estado ocorrem em episódios de Gradiente Negativo (Positivo) e/ou La Niña (El Niño). Estudos mostram que o efeito combinado do Gradiente do Atlântico e do El Niño oscilação Sul (ENOS) interferem diretamente na distribuição da pluviometria regional sobre a Amazônia oriental por meio do deslocamento da célula de Walker e/ou influenciando a posição da Zona de Convergência Intertropical (ZCIT) (De Souza et al., 2005; Andreoli e Kayano, 2006; Harris et al., 2008; Grimm e Zilli, 2009; Grimm e Tedeschi, 2009; Grimm, 2011; Coelho et al., 2012).

\subsection{Regiões homogêneas do SPI-6 na Amazônia Oriental}

A Fig. 4 ilustra a espacialização das regiões homogêneas obtidas a partir da aplicação do método de Ward aos principais fatores comuns espaciais do SPI-6 na Amazônia Oriental. Por meio deste procedimento identificaram-se três grupos, ou seja, três Regiões homogêneas do SPI-6 (doravante referida como RH): RH-1 localizada no nordeste do estado, possuindo 26 estações; RH-2 na porção central e noroeste, ocupando a maior área do Estado, com 32 estações e a RH-3 ao sul composta por 14 estações. Este resultado se assemelham com os obtidos por Amanajás e Braga (2012), onde foram identificados quatro grupos homogêneos de precipitação na Amazônia Oriental por meio do método aglomerativo de Ward.

Nas Tabelas 3 a 8 encontram-se as características (início, fim, duração e índice médio) dos principais eventos secos e chuvosos, com duração igual ou superior a 6 meses,

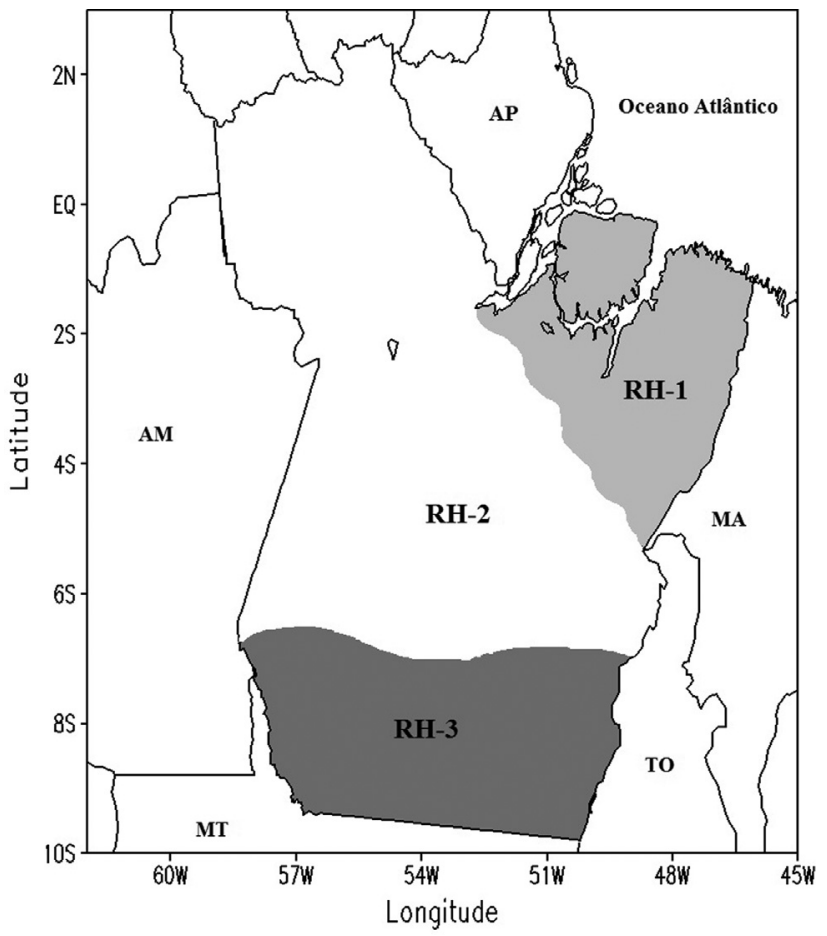

Figura 4 - Regiões homogêneas do SPI-6 no estado do Pará, obtidas a partir dos 16 fatores comuns espaciais mais significativos pelo método hierárquico de classificação de Ward.

em cada Região homogênea do SPI-6 a qual será discutida a seguir.

As Tabelas 3 e 4 ilustram as sequências de períodos secos e chuvosos para o SPI-6 na cidade de Castanhal, a qual representa o RH-1 do SPI-6. Foram identificados 15 eventos secos e 10 chuvosos. Verifica-se que a seca mais intensa ocorreu entre abril de 1993 a janeiro de 1994, cujo SPI médio foi igual a $-1,44$. Neste período ocorreu um evento de El Niño, como pode ser verificado nos resultados de Andreoli e Kayano (2006). Entretanto, a duração maior

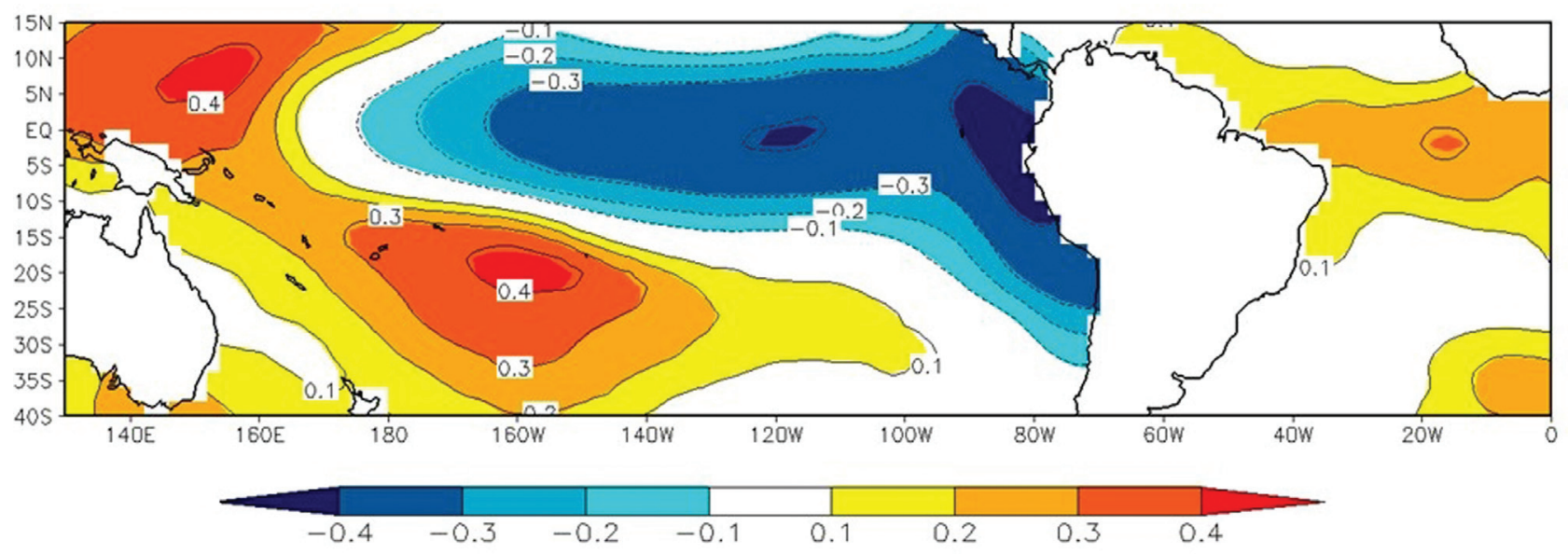

Figura 3 - Correlação entre série temporal (escore fatorial) do primeiro fator espacial do SPI-6 com as anomalias de TSM. A correlação apresentou 90\% de significância estatística pelo teste t-student. As anomalias de TSM foram calculadas considerando a climatologia para o período de junho de 1980 a dezembro de 2011. 
Tabela 3 - Características dos principais eventos de seca obtidas pelo SPI-6 em Castanhal no período de 1980-2011. Em negrito estão assinaladas as informações referentes aos extremos mais expressivos no período em estudo.

\begin{tabular}{lccccc}
\hline Inicio & Fim & Duração (meses) & SPI mínimo & SPI médio & Tipo de evento \\
\hline Jan/81 & Ago/82 & 20 & $-1,94$ & $-0,88$ & Seca Fraca \\
Out/82 & Jul/83 & 10 & $-2,70$ & $-1,42$ & Seca Moderada \\
Abr/85 & Out/85 & 7 & $-0,56$ & $-0,32$ & Quase Normal \\
Fev/87 & Dez/87 & 11 & $-0,80$ & $-0,45$ & Quase Normal \\
Abr/90 & Set/90 & 6 & $-0,89$ & $-0,33$ & Quase Normal \\
Out/91 & Jun/92 & 9 & $-1,97$ & $-0,59$ & Seca Fraca \\
Ago/92 & Fev/93 & 7 & $-2,25$ & $-1,31$ & Seca Moderada \\
Abr/93 & Jan/94 & $\mathbf{1 0}$ & $-\mathbf{2 , 9 9}, \mathbf{4 4}$ & Seca Moderada \\
Set/95 & Mar/97 & 19 & $-1,70$ & $-0,98$ & Seca Fraca \\
Fev/98 & Fev/99 & 13 & $-0,78$ & $-0,37$ & Quase Normal \\
Mar/00 & Out/00 & 7 & $-0,94$ & $-0,65$ & Seca Fraca \\
Jan/03 & Jun/03 & 6 & $-0,56$ & $-0,19$ & Quase Normal \\
Out/04 & Nov/05 & 14 & $-1,21$ & $-0,47$ & Quase Normal \\
Nov/06 & Ago/08 & $\mathbf{2 2}$ & $\mathbf{- 1 , 2 6}$ & Seca Fraca \\
Mar/10 & Ago/10 & 6 & $-1,51$ & $-0,84$ & Seca Fraca \\
\hline
\end{tabular}

Tabela 4 - Idem a Tabela 3, porém para os principais eventos de chuva.

\begin{tabular}{lccccc}
\hline Inicio & Fim & Duração (meses) & SPI máximo & SPI médio & Tipo de evento \\
\hline Out/84 & Mar/85 & 6 & 0,66 & 0,33 & Quase Normal \\
Nov/85 & Jan/87 & 15 & 1,03 & 0,61 & Chuva Fraca \\
Jan/88 & Mar/90 & $\mathbf{2 7}$ & $\mathbf{1 , 6 6}$ & $\mathbf{1 , 0 1}$ & Chuva Moderada \\
Abr/91 & Set/91 & 6 & 0,51 & 0,28 & Quase Normal \\
Fev/94 & Ago/95 & $\mathbf{1 9}$ & $\mathbf{3 , 1 9}$ & 0,64 & Chuva Severa \\
Abr/97 & Set/97 & 6 & 0,40 & 0,25 & Chuva Normal \\
Out/00 & Set/02 & 24 & 2,87 & 1,62 & Chuva Severa \\
Jul/03 & Set/04 & 15 & 0,67 & 0,29 & Quase Normal \\
Mai/09 & Fev/10 & 10 & 2,15 & 1,14 & Chuva Moderada \\
Set/10 & Abr/11 & 8 & & 0,62 & Quase Normal \\
\hline
\end{tabular}

Tabela 5 - Características dos principais eventos de seca obtidas pelo SPI-6 em Uruara no período de 1980-2011. Em negrito estão assinaladas as informações referentes aos extremos mais expressivos no período em estudo.

\begin{tabular}{lccccc}
\hline Inicio & Fim & Duração (meses) & SPI mínimo & SPI Médio & Tipo de evento \\
\hline Fev/81 & Dez/81 & 11 & $-1,56$ & $-0,79$ & Seca Fraca \\
Ago/82 & Fev/84 & $\mathbf{1 9}$ & $\mathbf{- 3 , 2 9}$ & $\mathbf{- 1 , 8 2}$ & Seca Severa \\
Mar/87 & Abr/88 & 14 & $-1,69$ & $-1,04$ & Seca Moderada \\
Fev/89 & Set/89 & 8 & $-0,72$ & $-0,51$ & Seca Fraca \\
Abr/90 & Out/90 & 7 & $-0,81$ & $-0,45$ & Quase Normal \\
Ago/91 & Jul/93 & $\mathbf{2 4}$ & $\mathbf{- 2 , 2 5}$ & $-\mathbf{1 , 0 0}$ & Seca Moderada \\
Jan/95 & Jun/95 & 6 & $-1,38$ & $-0,55$ & Seca Fraca \\
Fev/97 & Dez/98 & 23 & $-1,87$ & $-0,81$ & Seca Fraca \\
Dez/01 & Set/02 & 10 & $-1,97$ & $-1,17$ & Seca Moderada \\
Dez/02 & Mai/03 & 6 & $-0,73$ & $-0,36 \quad$ Quase Normal \\
\hline
\end{tabular}


Tabela 6 - Idem a Tabela 5, porém para os principais eventos de chuva.

\begin{tabular}{|c|c|c|c|c|c|}
\hline Inicio & Fim & Duração (meses) & SPI máximo & SPI médio & Tipo de evento \\
\hline Jan/82 & $\mathrm{Jul} / 82$ & 7 & 0,76 & 0,58 & Chuva Fraca \\
\hline $\mathrm{Mar} / 84$ & $\mathrm{Mar} / 85$ & 13 & 1,38 & 0,77 & Chuva Fraca \\
\hline Out/85 & Mai/86 & 8 & 1,82 & 0,93 & Chuva Fraca \\
\hline Mai/88 & Out $/ 88$ & 6 & 0,91 & 0,55 & Chuva Fraca \\
\hline Out/89 & $\mathrm{Mar} / 90$ & 6 & 1,02 & 0,68 & Chuva Fraca \\
\hline Out/93 & Dez/94 & 15 & 1,95 & 1,09 & Chuva Moderada \\
\hline Jan/96 & $\mathrm{Jan} / 97$ & 13 & 1,46 & 0,88 & Chuva Fraca \\
\hline Jan/99 & Ago/99 & 8 & 0,68 & 0,40 & Quase Normal \\
\hline $\mathrm{Abr} / 00$ & Jun/01 & 15 & 1,50 & 0,76 & Chuva Fraca \\
\hline Jun/03 & $\mathrm{Fev} / 04$ & 9 & 0,54 & 0,25 & Quase Normal \\
\hline Jun/04 & $\mathrm{Jul} / 05$ & 14 & 0,71 & 0,37 & Quase Normal \\
\hline Nov/05 & Dez/06 & 14 & 2,08 & 1,29 & Chuva Moderada \\
\hline Nov/07 & Out $/ 08$ & 12 & 1,25 & 0,95 & Quase Fraca \\
\hline Dez/08 & $\mathrm{Jul} / \mathbf{1 0}$ & 20 & 1,83 & 0,97 & Quase Fraca \\
\hline Nov/10 & $\mathrm{Jul} / 11$ & 9 & 1,23 & 0,82 & Chuva Fraca \\
\hline
\end{tabular}

Tabela 7 - Características dos principais eventos de seca obtidas pelo SPI-6 em Barreira do Campo no período de 1980-2011. Em negrito estão assinaladas as informações referentes aos extremos mais expressivos no período em estudo.

\begin{tabular}{lccccc}
\hline Inicio & Fim & Duração (meses) & SPI mínimo & SPI médio & Tipo de evento \\
\hline Set/81 & Fev/82 & 6 & $-1,49$ & $-0,59$ & Seca Fraca \\
Jan/83 & Set/83 & 9 & $-1,01$ & $-0,72$ & Seca Fraca \\
Set/86 & Ago/87 & 12 & $-0,68$ & $-0,42$ & Quase Normal \\
Mai/88 & Nov/88 & 7 & $-0,87$ & $-0,44$ & Quase Normal \\
Dez/90 & Mai/91 & 7 & $-0,68$ & $-0,39$ & Quase Normal \\
Jan/93 & Ago/93 & 8 & $-1,55$ & $-0,91$ & Seca Fraca \\
Mar/94 & Abr/95 & 14 & $-1,34$ & $-0,78$ & Seca Fraca \\
Set/95 & Out/98 & $\mathbf{3 8}$ & $-\mathbf{3 , 5 0}$ & $-\mathbf{1 , 6 6}$ & Seca Severa \\
Jan/99 & Fev/00 & 12 & $-2,12$ & $-0,78$ & Seca Fraca \\
Abr/07 & Out/08 & 19 & $-1,55$ & $-0,75$ & Seca Fraca \\
\hline
\end{tabular}

Tabela 8 - Idem a Tabela 7, porém para os principais eventos de chuva.

\begin{tabular}{lccccc}
\hline Inicio & Fim & Duração (meses) & SPI máximo & SPI médio & Tipo de evento \\
\hline Jun/80 & Mai/81 & 12 & 1,18 & 0,50 & Chuva Fraca \\
Mar/82 & Dez/82 & 10 & 1,33 & 0,45 & Quase Normal \\
Out/83 & Out/85 & $\mathbf{2 5}$ & $\mathbf{1 , 6 7}$ & $0, \mathbf{8 0}$ & Chuva Fraca \\
Mar/86 & Ago/86 & 6 & 0,94 & 0,65 & Chuva Fraca \\
Ago/89 & Mar/90 & 8 & 1,32 & 0,70 & Chuva Fraca \\
Jun/91 & Jul/92 & 14 & 0,97 & 0,32 & Quase Normal \\
Set/93 & Fev/94 & 6 & 1,03 & 0,59 & Chuva Fraca \\
Fev/00 & Set/00 & 8 & 1,19 & 0,75 & Chuva Fraca \\
Out/01 & Out/02 & 13 & 0,99 & 0,55 & Chuva Fraca \\
Dez/02 & Jun/03 & 7 & 0,42 & 0,26 & Quase Normal \\
Ago/03 & Ago/04 & 13 & 1,17 & $\mathbf{2}$ & Chuva Fraca \\
Out/04 & Set/05 & $\mathbf{1 2}$ & 2,07 & 1,04 & Chuva Moderada \\
Nov/05 & Mar/07 & 17 & 2,02 & Chuva Moderada \\
Nov/08 & Jun/10 & 20 & 2,94 & Chuva Fraca \\
\hline
\end{tabular}


de eventos secos foi de 22 meses (novembro de 2006 a agosto 2008). Os meses com eventos severos de chuva ocorreram entre fevereiro de 1994 a agosto de 1995, com duração de 19 meses e SPI-6 médio igual a 1,64. De janeiro de 1988 a março de 1990 houve 27 meses consecutivos de chuva com intensidade moderada, sob influência do fenômeno La Niña (Andreoli e Kayano, 2006). Na análise do histograma de frequência, observou-se 7 ocorrências de extremo de seca, 18 de seca severa, 21 de seca moderada, 67 de seca fraca, 169 de eventos quase normais, 46 de chuva fraca, 21 de chuva moderada, 10 de chuva severa e 20 de chuva extrema (Fig. 5). Para esta região do SPI-6, constata-se um maior número de meses consecutivos secos, porém, com pequena durabilidade.

Verifica-se nas Tabelas 5 e 6, as principais características do SPI-6 para a cidade de Uruara, que representa a RH-2 do SPI-6. O maior período de meses secos foi de 24 meses, que ocorreu entre agosto de 1991 a julho de 1993, além disso foi registrado uma seca severa com 19 messes, no período de agosto de 1982 a fevereiro de 1984, com média de SPI-6 igual a -1,82. Segundo Ronchail et al. (2005) e Marengo (2008) nos anos de 1982 a 1984 houve alguns episódios de El Niño combinados com o aquecimento anômalo do Oceano Atlântico tropical Norte, sugerindo que estes fenômenos diminuíram o regime de chuvas nesta região.

A chuva moderada mais intensa ocorreu no período de novembro de 2005 a dezembro de 2006 com SPI-6 médio igual a 1,29 , seguido de um evento moderado de menor intensidade entre outubro de 1993 a dezembro de 1994. De acordo com Andreoli e Kayano (2006), neste período houve episódios de El Niño fraco, o que pode ter influenciado, mesmo em menor intensidade as chuvas semestrais desse grupo.

A Fig. 6 ilustra distribuições das frequências das 9 categorias de eventos para a cidade de Uruara. Para essa região homogênea, registraram-se ocorrências de: 15 eventos de seca extremas, 17 de secas severas, 28 de secas moderados, 43 de seca fraca, 152 eventos quase normais, 65 de chuva fraca, 42 de chuva moderada, 16 eventos

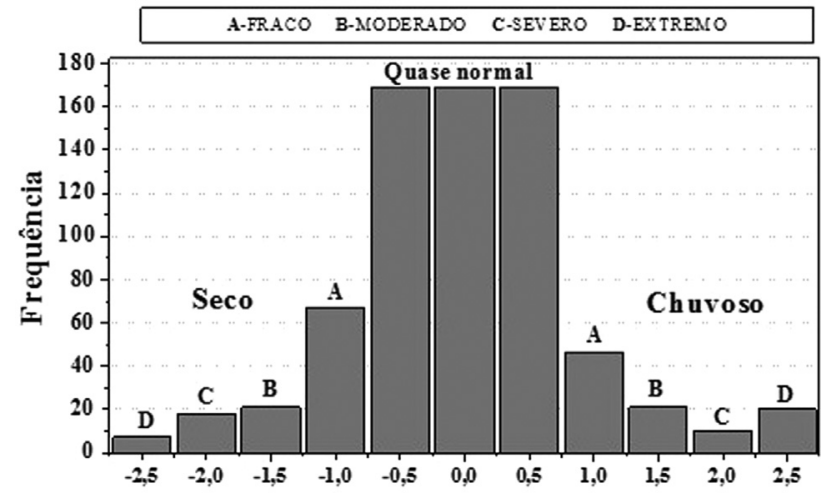

Figura 5 - Distribuições de frequência para as 9 categorias do SPI em Castanhal localizada na RH-1 do SPI-6, no estado do Pará.

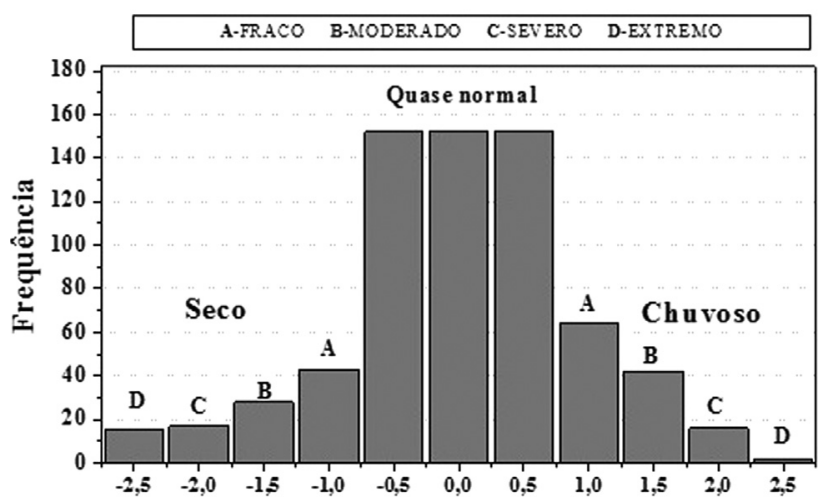

Figura 6 - Distribuições de frequência para as 9 categorias do SPI em Uruara localizada na RH-2 do SPI-6, no estado do Pará.

severo de chuva e 2 eventos extremo de chuva. Nesta região, não foram detectados eventos de chuvas severas e nem extremas, apenas moderada e fraca.

$\mathrm{Na}$ cidade de Barreira do Campo, representativa da RH-3 do SPI-6 ocorreram 10 eventos secos e 12 eventos chuvosos. A seca mais severa registrada no período em estudo, com duração de 38 meses e SPI-6 médio igual a -1,66, ocorreu entre setembro de 1995 a outubro de 1998 (Tabela 7). Espinoza et al. (2011) observaram neste período episódios de fenômenos El Niño, além disso os autores constataram o aquecimento anômalo do Oceano Atlântico Tropical Norte. O período de chuvas mais intensas foi em outubro de 2004 a setembro de 2005 com SPI médio igual a 1,28. Em termos médios, não foram registrados eventos severos nem extremos de chuvas neste grupo, prevalecendo a classe de eventos fracos de chuva (Tabela 8).

A distribuição das frequências referente ao SPI-6 para a cidade de Barreira do Campo apresenta 15 ocorrências de seca extrema, 15 de seca severa, 23 de seca moderada, 44 de seca fraca, 169 eventos quase normais, 61 de chuva fraca, 35 de chuva moderada, 13 de chuva severa e 4 de chuva extrema (Fig. 7). Observa-se pelas distribuições de frequências dos anos analisados nas 3 décadas, que a RH-3

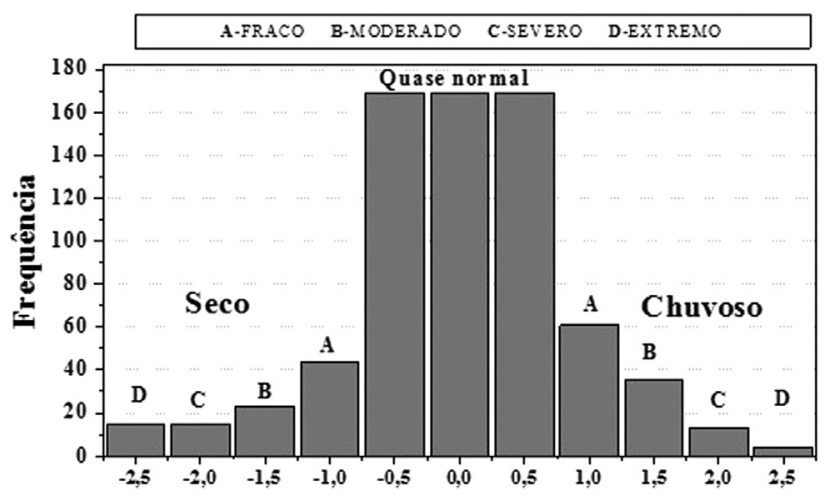

Figura 7 - Distribuições de frequência para as 9 categorias do SPI em Barreira do Campo localizada na RH-3 do SPI-6, no estado do Pará. 
registrou os mais longo e intensos períodos de secas semestrais, quando comparado com as demais RH.

\section{Conclusões}

A aplicação da ACP e Agrupamentos aos dados do SPI-6 identificou 3 Regiões Homogêneas. A RH-1 localizada no nordeste do estado apresentou 15 eventos secos e 10 de chuva. Na RH-2 que ocupa a maior parte do estado observou-se 10 eventos secos e 15 de chuva. E a RH-3 situada ao sul apresentou 10 eventos secos e 15 chuvosos para o período estudado.

De modo geral, a região nordeste possui mais eventos secos que chuvosos. Nas demais regiões (central e sul) a situação é inversa, isto é, existem mais eventos de chuva, porém de pouca duração. Adicionalmente, a regão sul do Estado apresentou a seca mais severa e prolongada. Além disso, é importante salientar que os eventos secos e chuvosos nas RH's estavam associados a variabilidade climática que ocorre nos oceanos Atlântico (Gradiente do Atlântico) e Pacifico (ENOS).

Com os resultados obtidos é possível, por exemplo, melhorar o zoneamento agrícola da Estado do Pará, informando que as regiões costeria e sul do Estado são mais suscetíveis a longos períodos de seca, e a porção mais central do Estado está mais suscetíveis a longos períodos de chuva. Assim, evitando nestas áreas plantio e/ou colheita em períodos com forte risco de ocorrência de chuvas e/ou secas e a consequente redução da qualidade da produção. $\mathrm{O}$ conhecimento científico apresentado neste trabalho também pode auxiliar na seleção das culturas de menor risco para estas regiões, bem como o estabelecimento das datas de plantio mais favoráveis nas mesmas.

\section{Agradecimentos}

O primeiro autor agradece ao Programa de Pós-Graduação em Meteorologia da Universidade Federal de Campina Grande pela concessão de bolsa de mestrado do CNPq e juntamente com os demais autores, agradecemos ao INMET e ANA pelos dados cedidos.

\section{Referências}

ANDREOLI, R.V.; KAYANO, M.T. Tropical Pacific and South Atlantic Effects on Rainfall Variability over Northeast Brazil. International journal of climatology, v. 26, n. 13, p. 1895-1912, 2006.

ACEITUNO, P. On the Functioning of the Southern Oscillation in the South American sector. Part I: Surface climate. Monthly Weather Review, v. 116, n. 3, p. 505-524, 1988.

AMBRIZZI, T.; ROCHA, R.; MARENGO, J.; PISNITCHENKO, L.; ALVES, F. Cenários Regionalizados de Clima no Brasil para o Século XXI: Projeções de clima usando três modelos regionais. Ministério do Meio Ambiente-MMA, Secretaria de Biodiversidade e Florestas-SBF, Diretoria de Conservação da Biodiversidade, 2007.
AMANAJÁS, J.C.; BRAGA, C.C. Padrões Espaço-Temporal Pluviométricos na Amazônia Oriental Utilizando Análise Multivariada. Revista Brasileira de Meteorologia, v. 27, n. 4, p. 423-434, 2012.

BONACCORSO, B.; BORDI, I.; CANCELLIERE, A.; ROSSI, G.; SUTERA, A. Spatial Variability of Drought: An Analysis of the SPI in Sicily. Water Resources Management, v. 17, n. 4, p. 273-296, 2003.

DE SOUZA, E.B.; KAYANO, M.T.; AMBRIZZI, T. Intraseasonal and submonthly variability over the eastern Amazon and Northeast Brazil during the autumn rainy season. Theoretical and Applied Climatology, v. 81, p. 177-191, 2005

DOS SANTOS, S.R.Q.; BRAGA, C.C.; SANTOS, A.P.P.; BRITO, J.I.B.; CAMPOS, T.L .O.B. Classificação de Eventos Extremos de Precipitação em Múltiplas Escalas de Tempo em Belém-PA: Utilizando o Índice de Precipitação Normalizada. Revista Brasileira de Geografia Física, v. 7, n. 4, p. 628-635, 2014.

DOS SANTOS, S.R.Q.; BRAGA, C.C.; CAMPOS, T.L.O.B.; BRITO, J.I.B.; SANTOS, A.P.P. Variabilidade da Precipitação no Estado do Pará por meio de Análise em Componentes Principais. Revista Brasileira de Geografia Física, v. 7, n. 3, p. 615-627, 2014.

DOS SANTOS, S.R.Q.; SILVA, R.B C.; BARRETO, P.N.; NUNES, H.G.G.C.; RODRIGUES, R.S.; CAMPOS, T.L.O.B. Regime Térmico e Hídrico do solo para área de Floresta Tropical em anos de El Niño e La Niña, Caxiuanã-PA: Estudo de Caso. Revista Brasileira de Meteorologia, v. 26, n. 3, p. 367-374, 2011.

CAVALCANTI, I.F.A. Large scale and synoptic features associated with extreme precipitation over South America: A review and case studies for the first decade of the 21 st century. Atmospheric Research , v. 118, p. 27-40, 2012.

CAVALCANTI, I.F.A.; KOUSKY, V.E. Drought in Brazil during Summer and Fall 2001 and associated atmospheric circulation features. Climanálise, 2004.

COX, P.; HARRIS, P.; HUNTINGFORD, C.; BETTS, R.; COLLINS, M.; CHRIS, J. ; JUPP, T.; MARENGO J.A.; NOBRE, C. Increase risk of Amazonian Drought due to decreasing aerosol pollution. Nature, v. 453, p. 212-216, 2008.

COELHO, C.A.S.; CAVALCANTI, I.F.A.; COSTA, S.M.S.; FREITAS, S.R.; ITO, E.R.; LUZ, G.; SANTOS, A.F.; NOBRE, C.A.; MARENGO, J.A.; PEZZA, A.B. Climate diagnostics of three major drought events in the Amazon and illustrations of their seasonal precipitation predictions. Meteorological Applications, v. 19, p. 237-255. 2012.

ESPINOZA, J.C.; RONCHAIL, J.; GUYOT, J.L.; JUNQUAS, C.; VAUCHEL, P.; LAVADO, W.; POMBOSA, R. Climate variability and extreme drought in the upper Solimões River (western Amazon Basin): Understanding the exceptional 2010 drought. Geophysical Research Letters, v. 38, n. 13, 2011.

ESPINOZA， J.C.; LENGAIGNE， M.; RONCHAIL， J.; JANICOT, S. Large-scale circulation patterns and related rainfall in the Amazon Basin: a neuronal networks approach. Climate dynamics, v. 38, n. 1-2, p. 121-140, 2012.

ESPINOZA, J.C.; MARENGO, J.A.; RONCHAIL, J.; CARPIO, J.M.; FLORES, L.N.; GUYOT, J.L. The extreme 2014 flood in south-western Amazon basin: the role of tropical-sub- 
tropical South Atlantic SST gradient. Environmental Research Letters, v. 9, n. 12, p. 124007, 2014.

EVERITT, B.S. Cluster analysis. Edward Arnold and Halsted Press, 1993.

GRIMM, A.M. Interannual climate variability in South America: impacts on seasonal precipitation, extreme events, and possible effects of climate change. Stochastic Environmental Research and Risk Assessment, v. 25, n. 4, p. 537-554, 2011.

GRIMM, A.M.; TEDESCHI, R.G. ENSO and extreme rainfall events in South America. Journal of Climate 22, 15891609, 2009

GRIMM, A.M.; ZILLI, M.T. Interannual variability and seasonal evolution of summer monsoon rainfall in South America. Journal of Climate, v. 22, n. 9, p. 2257-2275, 2009

GUTTMAN, N.B. Comparing the Palmer drought index and the standardized precipitation index. Journal of the American Water Resources Association, v. 34, n. 1, p. 113-121, 1998.

GONG, X.; RICHMAN, M.B. On the application of cluster analysis to growing season precipitation data in North America east of the Rockies. Journal of Climate, v. 8, n. 4, p. 897-931, 1995

HAYES, M.J. Revisiting the SPI: clarifying the process. 2000.

LOPES, M.N.G.; SOUZA, E.B.; FERREIRA, D.B.S. Climatologia Regional da Precipitação no Estado do Pará. Revista Brasileira de Climatologia, v. 12, p. 84-102, 2013.

MARENGO, J.A. Mudanças climáticas globais e seus efeitos sobre a biodiversidade: caracterização do clima atual e definição das alterações climáticas para o território brasileiro ao longo do século XXI. MMA, Ministério do Meio Ambiente, 2006.

MARENGO, J.A.; ALVES, L.; VALVERDE, M.; ROCHA, R.; LABORBE, R. Eventos extremos em cenários regionalizados de clima no Brasil e América do Sul para o Século XXI: Projeções de clima futuro usando três modelos regionais. Relatório, v. 5, p. 495-516, 2007.

MARENGO, J.A.; NOBRE, C.; TOMASELLA, J.; OYAMA, M.; SAMPAIO, G.; CAMARGO, H.; ALVES, L.M. The drought of Amazonia in 2005. Journal of Climate, v. 21, p. 495-516, 2008.

MARENGO, J.A.; TOMSASELLA, J; ALVES, L.M.; SOARES, W.; RODRIGUEZ, D.A. The drought of 2010 in the context of historical droughts in the Amazon region. Geophysical Research Letters, v. 38, p. 1-5, 2011.

MARENGO, J.A. Mudanças Climáticas, Condições Meteorológicas extremas e eventos Climáticos No Brasil. Fundação Brasileira para o Desenvolvimento Sustentável. Disponível em: http://www.fbds.org.br/cop15/FBDS_MudancasClimaticas .pdf. 2012.

MARENGO, J.A.; TOMASELLA, J.; SOARES, W.R.; ALVES, L.M.; NOBRE, C.A. Extreme climatic events in the Amazon basin. Theoretical and Applied Climatology, v. 107, n. 1-2, p. 73-85, 2012.

MARENGO, J.A.; BORMA, L.S.; RODRIGUEZ, D.A.; PINHO, P.; SOARES, W.R.; ALVES, L.M. Recent extremes of drought and flooding in Amazonia: vulnerabilities and human adaptation. 2013.

MCKEE, T.B.; DOESKEN, N.J.; KLEIST, J. The relationship of drought frequency and duration to time scales. In: Proceed- ings of the 8th Conference on Applied Climatology. American Meteorological Society, p. 179-183, 1993.

MICHAELIDES, S.; PASHIARDIS, S. Monitoring drought in Cyprus during the 2007-2008 hydrometeorological year by using the standardized precipitation index (SPI). European Water, v. 23, n. 24, p. 123-131, 2008.

NORTH, G.R.; BELL, T.L.; CAHALAN, R.F.; MOENG, F.J. Sampling errors in the estimation of empirical orthogonal functions. Monthly Weather Review, v. 110, n. 7, p. 699-706, 1982.

NING ZENG; YOON, J.; MARENGO J.A.; SUBRAMANIAM, A.; NOBRE, C.; MARIOTTI, A. Causes and impacts of the 2005 Amazon drought. Environmental Research (New York), v. 3, p. 1-6, 2008.

Painel Brasileiro de Mudanças Climáticas - PBMC- Disponível em http://www.brasil.gov.br/meio-ambiente/2012/04/ mudancas-climaticas Consultado em: 10 de junho de 2015.

RICHMAN, M.B. Rotation of principal components. Journal of Climate, v. 6, n. 3, p. 293-335, 1986.

RONCHAIL, J.; BOURREL, L.; COCHONNEAU, G.; VAUCHEL, P.; PHILLIPS, L.; CASTRO, A.; DE OLIVEIRA, E. Inundations in the Mamoré basin (south-western Amazon-Bolivia) and sea-surface temperature in the Pacific and Atlantic Oceans. Journal of Hydrology, v. 302, n. 1, p. 223-238, 2005.

RAO, D.U.M.; REDDY, T.S.; REDDY, G.R. Atmospheric radar signal processing using principal component analysis. Digital Signal Processing, v. 32, p. 79-84, 2014.

MICHAEL, B.R. Rotation of principal components. Journal of climatology, v. 6, p. 293-335, 1986.

SANTOS, C.A.C.; SATYAMUITY, P.; SANTOS, E.M. Tendências de índices de extremos climáticos para a região de Manaus-AM. Acta Amazonica, v. 42, p. 329-336, 2012.

SANTOS, C.A.C. Recent changes in temperature and precipitation extremes in an ecological reserve in Federal District, Brazil. Revista Brasileira de Meteorologia, v. 29, p. 13-20, 2014.

SÖNMEZ, F.K.; KÖMÜSCÜ, A.Ü.; ERKAN, A.; TURGU, E. An analysis of spatial and temporal dimension of drought vulnerability in Turkey using the standardized precipitation index. Natural Hazards, v. 35, n. 2, p. 243-264, 2005.

SADEGHI, L.; SHAMSELDIN, A.Y. Application of the Standardized Precipitation Index (SPI) in Hawke's Bay, New Zealand. Drought: Research and Science-Policy Interfacing, p. 139, 2014.

SANSIGOLO, C.A. Análise comparativa do desempenho dos Índices de Seca de Palmer (PDSI) e da Precipitação Normalizada (SPI) em Piracicaba, SP (1917-2001). Revista Brasileira de Meteorologia, v. 19, n. 1, p. 51-56, 2004.

SIMIELLI, M.E. Geoatlas. São Paulo: Editora Ática, 1991

TOMASELLA, J.; BORMA, L.S.; MARENGO, J.A. RODRIGUEZ, D.A.; CUARTAS, L.A.; NOBRE, C.; PRADO, M.C.R. The droughts of 1996-1997 and 2004-2005 in Amazonia: hydrological response in the river main-stem. Hydrological Processes, v. 25, p. 1228-1242, 2011.

THOM, H.C.S. A note on the gamma distribution. Monthly Weather Review, v. 86, n. 4, p. 117-122, 1958.

UVO, C.B.; REPELLI, C.A.; ZEBIAK, S.E.; KUSHNIR, Y. The relationships between tropical Pacific and Atlantic SST and northeast Brazil monthly precipitation. Journal of Climate, v. 11, n. 4, p. 551-562, 1998. 
WARD, J.H. Hierarchical grouping to optimize an objective function. Journal of the American statistical association, v. 58, n. 301, p. 236-244, 1963.

WILKS, D.S. Statistical Methods in the Atmospheric Sciences Academic. 2006.

WILLIAMS, E.; DALL'ANTONIA, A.; DALL'ANTONIA, V.; ALMEIDA, J.M.D.; SUAREZ, F.; LIEBMANN, B.; MALHADO, A.C.M. The drought of the century in the Amazon Basin: An analysis of the regional variation of rainfall in South America in 1926. Acta Amazonica, v. 35, n. 2, p. 231-238, 2005.
ZHANG, Q.; XU, C.; ZHANG, Z. Observed changes of drought/wetness episodes in the Pearl River basin, China, using the standardized precipitation index and aridity index. Theoretical and Applied Climatology, v. 98, n. 1-2, p. 89-99, 2009.

ZENG, N.; YOON, J.H.; MARENGO, J.A.; SUBRAMANIAM, A.; NOBRE, C.A.; MARIOTTI, A.; NEELIN, J.D. Causes and impacts of the 2005 Amazon drought. Environmental Research Letters, v. 3, n. 1, p. 014002, 2008.

This is an Open Access article distributed under the terms of the Creative Commons Attribution Non-Commercial License which permits unrestricted non-commercial use, distribution, and reproduction in any medium provided the original work is properly cited. 